\title{
Numerical Simulations of Serrated Propellers to Reduce Noise
}

\author{
Wee-beng Tay ${ }^{1(\triangle)}(\mathbb{D})$, Zhenbo Lu ${ }^{1}(\mathbb{D})$, Sai Sudha Ramesh ${ }^{1}(\mathbb{D})$, \\ and Boo-cheong $\mathrm{Khoo}^{2}$ (D) \\ 1 Temasek Laboratories, National University of Singapore, T-Lab Building, \\ 5A, Engineering Drive 1, \#02-02, Singapore 117411, Singapore \\ tsltaywb@nus.edu.sg \\ 2 Department of Mechanical Engineering, National University of Singapore, \\ 10 Kent Ridge Crescent, Singapore 119260, Singapore
}

\begin{abstract}
The objective of this research is to investigate the effect of serrations on quadcopter propeller blades on noise reduction through numerical simulations. Different types of the 5 inch 5030 propellers, such as the standard, modified and serrated, are tested. The modified propeller has a portion of its blade's trailing edge cut off to achieve the same surface area as that of the serrated blades to ensure a fairer comparison. Three-dimensional simulations propellers have been performed using an immersed boundary method (IBM) Navier-Stokes finite volume solver to obtain the velocity flow fields and pressure. An acoustic model, based on the well-known Ffowcs WilliamsHawkings (FW-H) formulation, is then used to predict the far field noise caused by the rotating blades of the propeller. Results show that due to the reduction in surface area of the propeller's blades, there is a drop in the thrust produced by modified and serrated propellers, compared to the standard one. However, comparing between the modified and serrated propellers with different wavelength, we found that certain wavelengths show a reduction in noise while maintaining similar thrust. This is because the serrations break up the larger vortices into smaller ones This shows that there is potential in using serrated propellers for noise reduction.
\end{abstract}

Keywords: Serrated trailing edge $\cdot$ Noise reduction $\cdot$ Propeller $\cdot$ Immersed boundary method

\section{Introduction}

Today, renewed attention is being focused on the first aeronautical propulsion device: the propeller. This is due to the increased use of unmanned air vehicles (UAVs), the growing market of general aviation, the increasing interest in ultralight categories or light sport air vehicles, and the growing importance of environmental issues that have led to the development of all-electric emissionless aircraft. One of the most popular small aircraft choices (weighing around 250-350 g) nowadays is the quadcopter, mostly in part due to its low cost, mechanical simplicity and versatile applications. However, one disturbing problem of propeller-driven aircrafts is their noise, which may 
limit the aircraft's operation. This can be a serious concern if a UAV wishes to remain tactical, especially indoors since the audible noise level indoors is much lower.

Reducing propeller's noise can be achieved by a systematic or novel design of the propeller's geometry and aerodynamic characteristics. Most of the research work has been directed towards conventional engineering strategies to achieve good propeller designs. For instance, the performance of propellers can be improved by adjusting the number of blades, diameter, airfoil shape/distribution, chord, pitch distribution and coning angle [1]. Another method is through the use of contra-rotating propellers [2].

Alternatively, we can look to nature for inspirations. In contrast to conventional engineering strategies, studies on the application of bio-inspired features in propeller designs have been initiated recently [3-5]. One example is the owls, which developed serrated feathers on their wings and downy feathers on their legs that minimize aerodynamic noise, giving them silent flight. The serrations give the owl a better ability to control airflow, therefore allowing it to fly faster and achieve noise reduction at the same time. Another bio-inspired design is the porous trailing edge [6]. Ziehl-Abegg, Inc. primarily a ventilator company harnessed this feature by adding winglets to the blade tip and creating a serrated trailing edge on the rotor blades for achieving a quiet axial fan (FE2owlet axial fan). This resulted in a significant noise reduction up to $12 \mathrm{dBA}$. However, due to the patent protection, only a few reference works related to this product can be found from the website. Thus, systematic research work for further developing a quiet UAV propeller system using this bio-propeller noise reduction concept is required.

The objectives of the present study are to preliminarily explore this bio-propeller concept using numerical modelling and further develop a low noise bio-propeller design strategy which can be used to optimize the propeller's blade geometry of the small $(<20 \mathrm{~cm})$ quadcopter. We will develop numerical models for calculating the aerodynamics and aero-acoustic performances of the propeller with focus on biomimetic serrated blades design using an in-house 3D Immersed Boundary Method (IBM) [7] Navier-Stokes solver, coupled with a Ffowcs Williams and Hawkings (FWH) [8] acoustic code. A systematic analysis will be performed to improve the aeroacoustic performance of a bio-inspired propeller with a tentative goal of reducing its acoustic signature. Lastly, experimental validation will be performed to ensure that the numerical simulations have been performed accurately.

\section{Numerical Setup}

\subsection{Aerodynamic Solver}

For our simulations, an immersed-boundary method (IBM) [7] Navier-Stokes numerical solver [9] is used in this study. The reason for using an IBM based solver is because the blades of the propeller rotate. In some standard grid conforming numerical solvers which use the Arbitrary Lagrangian-Eulerian (ALE) [10] formulation, there is a need to constantly perform grid deformation or remeshing due to the blades' rotation.

This slows down the solver and affects the quality of the solution. A workaround is to enclose the propeller in a cylindrical domain and rotate that entire domain. However, 
there is also another problem with regards to the serrated propellers, as it is not trivial creating meshes in the vicinity of the serrations on the blades.

On the other hand, in IBM, the entire domain is composed of Cartesian grid and our bodies of interest are "immersed" in this grid, as shown in Fig. 1. To simulate the presence of the bodies, we need to add an additional forcing term $f c$ to the momentum equation to give:

$$
\frac{\partial u}{\partial t}=-u \cdot \nabla u+\frac{1}{\operatorname{Re}} \nabla^{2} u-\nabla p+f c
$$

where $u$ is the velocity vector, $t$ is the time, $p$ is the pressure and Re is the Reynolds number. Equation (1) has been non-dimensionalized using the blade's velocity $\left(U_{r e f}\right.$, at distance of $75 \%$ from its root) and mean chord length $(c)$ as the reference velocity and length respectively.

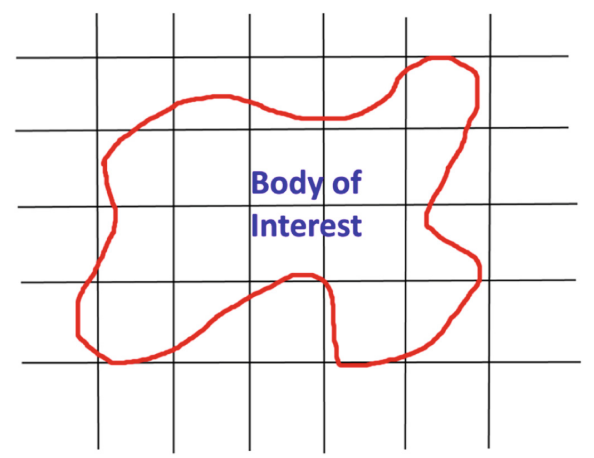

Fig. 1. Body of interest immersed inside Cartesian grid.

Out of the different variants of IBM, the discrete forcing approach is chosen because it is more suitable for our current Reynolds number (Re) of 31,407. This approach is based on a combination of the methods developed by Yang and Balaras [11], Kim et al. [12] and Liao et al. [13]. In the scheme, $f c$ is provisionally calculated explicitly using the $1^{\text {st }}$ order forward Euler and $2^{\text {nd }}$ order Adams Bashforth (AB2) schemes for the viscous and convective terms, respectively, to give:

$$
f c^{n+1}=\frac{u_{f}-u^{n}}{\Delta t}+\left(\frac{3}{2} \nabla \cdot(u u)^{n}-\frac{1}{2} \nabla \cdot(u u)^{n-1}\right)-\left(\frac{\nabla^{2} u}{\operatorname{Re}}\right)^{n}+\nabla p^{n},
$$

where $n$ refers to the time step.

$$
\nabla \cdot u=0
$$

Equation (3) is the continuity equation. To solve the modified non-dimensionalized incompressible Navier-Stokes equations (Eq. (1) and Eq. (3)), the finite volume 
fractional step method, based on an improved projection method, is used. For the time integration, the second order AB2 and Crank Nicolson (CN2) discretization are used for the convective and viscous terms, respectively. For the spatial derivatives, the convective and viscous terms are discretized using the second order central differencing on a staggered grid. We solve Eq. (1) and (3) using the fractional step method as described by Kim and Choi [14], whereby the momentum equation is first solved to obtain a non-divergence free velocity field. Using this non-divergence free velocity, we solve the Poisson equation to obtain the pressure field, which in turn updates the velocity to be divergence free. The open source linear equation solvers PETSc [15] and HYPRE [16] are used to solve the momentum and Poisson equations respectively. At this relatively low Re of 31,407 , no turbulence modelling is necessary because the flow is still largely laminar.

\subsection{Force Calculations}

Due to the fact that the body is not aligned with the Cartesian grid in the IBM, the forces acting on the bodies are calculated in a different way, as compared to the standard grid conforming solvers. In this case, we use the forcing term $f c_{n+1}$ obtained earlier to calculate the non-dimensional force $F_{i}$ on the body. More details about this method can be found in the paper by Lee et al. [15]:

$$
F_{i}=-\int_{\text {solid }} f c_{i}^{n+1} d V+\int_{\text {solid }}\left(\frac{\partial u_{i}}{\partial t}+\frac{\partial u_{i} u_{j}}{\partial x_{j}}\right) d V
$$

where $V$ is the volume of the wing.

The thrust coefficients $c_{t}$ is then given by:

$$
c_{t}=\frac{2 c^{2} F_{t}}{S}
$$

where $V$ is the volume of the wing.

The thrust coefficients $c_{t}$ is then given by:

$$
c_{t}=\frac{2 c^{2} F_{t}}{S}
$$

where $c$ and $S$ refer to the reference wing mean chord length and wing surface area, respectively.

\subsection{Solver Validation}

The current IBM solver has been validated many times with different experiments. Some of the examples are:

1. Plunging wing placed in a water tunnel at a $\mathrm{Re}$ of 10,000 with an angle of attack of $20^{\circ}[17]$ 
2. Simultaneous sweeping and pitching motion of a hawkmoth-like wing in a water tunnel at a $\operatorname{Re}$ of 10,000 [18].

More details about the validation can be found in the paper by Tay et al. [9].

\subsection{Acoustic Solver}

We use a permeable form of FW-H equation, wherein the integration surface (a fictitious control surface) surrounds the non-linear flow region. This enables representation of the non-linear flow effects through the surface source terms in the equation [19]. The fictitious control surface onto which the CFD flow variables are projected, is assumed to be stationary. In the present study, the permeable control surface onto which the CFD flow variables (namely, pressure and velocity components) are projected, is assumed to be stationary. Hence, for a stationary control surface with negligible density fluctuations, the solution for acoustic pressure is given as follows:

$$
\begin{aligned}
& 4 \pi p^{\prime}(x, t)=\int_{S}\left[\frac{\rho_{0} \dot{u}_{n}}{r}\right]_{\tau} d S(y)+\int_{S}\left[\frac{\dot{p} \hat{n} \cdot \hat{r}}{c_{0} r}\right]_{\tau} d S(y)+ \\
& \int_{S}\left[\frac{p \hat{n} \cdot \hat{r}}{r^{2}}\right]_{\tau} d S(y)+\int_{S}\left[\frac{\rho_{0}\left(u_{n} u_{r}\right)}{c_{0} r}\right]_{\tau} d S(y)+\int_{S}\left[\frac{\rho_{0} u_{n} u_{r}}{r^{2}}\right]_{\tau} d S(y)
\end{aligned}
$$

where $\rho_{0}$ denotes the ambient fluid density; $c_{0}$ is the speed of sound; $u_{n}$ denotes the dot product of the velocity vector with the unit normal vector $\hat{n} ; \tau$ refers to the source time and $t$ is the observer time given as $t=\tau+\left(r / c_{0}\right) ; y$ denotes the source location; $r$ denotes the source observer distance. The subscripts $n$ and $r$ denote dot products with the unit vectors in the normal $\hat{n}$ and radiation $\hat{r}$ directions respectively. The Farassat $1 \mathrm{~A}$ formulation has been used to transfer the time derivatives in the observer time into the surface integral terms in the FW-H equation, in order to prevent numerical instabilities. This results in a retarded-time formulation, which is solved using a mid-panel quadrature method and a source time-dominant algorithm [20]. Once the observer time pressure history is obtained, a fast Fourier transform (FFT) of the time series is performed to obtain the sound pressure level in frequency domain.

\subsection{Simulation Setup and Grid Convergence Study}

In this study, the reference velocity $U_{\infty}$ is chosen as the tangential velocity $75 \%$ of the blade length from the propeller's root, which is calculated to be $44.77 \mathrm{~m} / \mathrm{s}$, with the blade length $=0.127 \mathrm{~m}$ and rotation speed $=9,000 \mathrm{rpm}$. The reference length is the average blade's chord length, which is $0.011 \mathrm{~m}$. This gives a $\operatorname{Re}$ of 31,407 . The reduced frequency is given as:

$$
f_{r}=\frac{f c}{U_{\infty}}=0.037
$$

where $f$ and $c$ are the frequency and chord length respectively.

Since the solver is IBM based, only Cartesian grids are used. The size of the computational domain is $24 \times 24 \times 25$ (in terms of non-dimensional chord length $c$ ) 
in the $x, y$ and $z$-directions respectively. The domain varies from -12 to $12,-12$ to 12 and 0 to 25 in the $x, y$ and $z$-directions respectively. The propeller is placed at the $x=0$, $y=0, z=6$ location. Refinement is used in the region near the propeller and this region consists of uniform grid cells of length $d x$, which is the minimum grid length and it gives an indication of the resolution of the overall grid. We perform the simulations in quiescent flow, similar to the experimental setup.

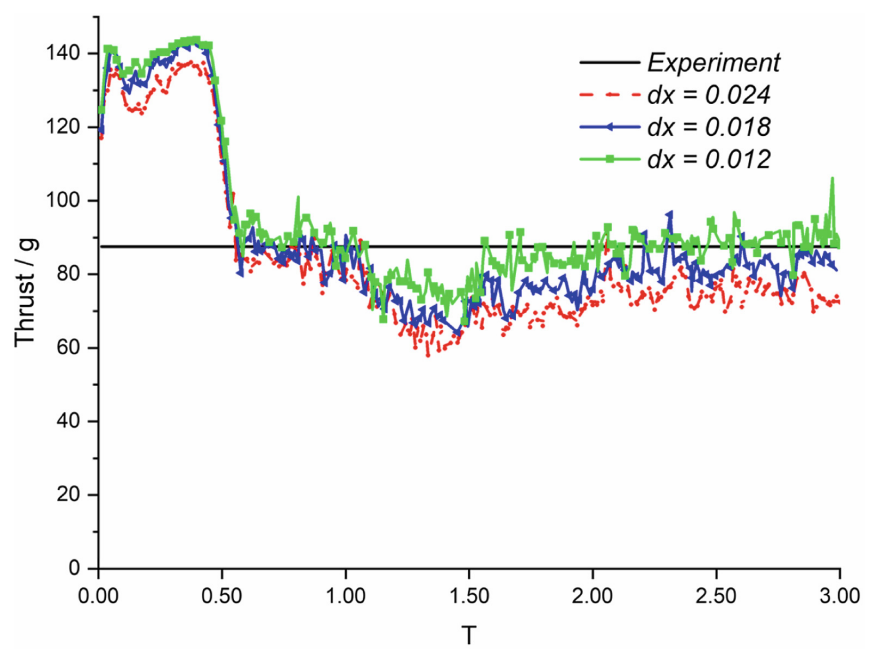

Fig. 2. Comparison of thrust with experiment and at $d x=0.024,0.018$ and 0.012 .

Table 1. Average thrust obtained by experiment and at at $d x=0.024,0.018$ and 0.012

\begin{tabular}{l|l|l|l|l}
\hline Avg thrust/g & Experiment & $d x=0.024$ & $d x=0.018$ & $d x=0.012$ \\
\cline { 2 - 5 } & 87.5 & 75.2 & 82.1 & 89.1 \\
\hline
\end{tabular}

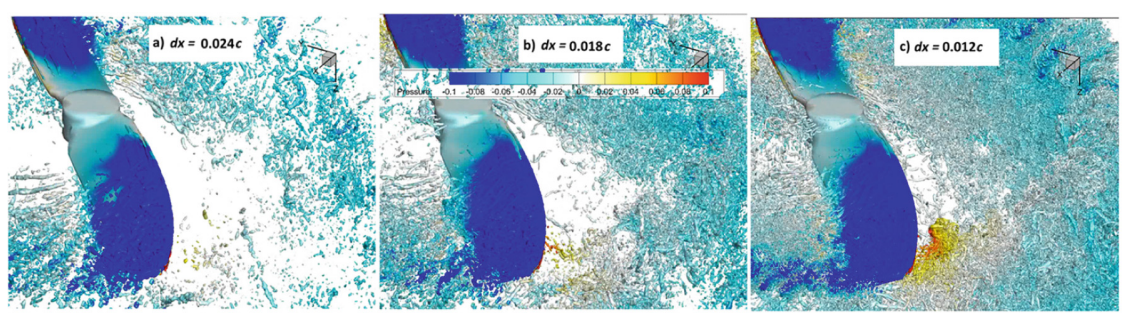

Fig. 3. Isosurfaces plotted at $\mathrm{Q}$ criterion $=2$ superimposed with pressure contour at time $=0.12 \mathrm{~T}$ with $d x=$ a) 0.024 , b) 0.018 and c) 0.012 . 
For the grid convergence study, we perform simulations at $d x=0.024 c, 0.018 c$ and $0.012 c$, which translate to total grid sizes of $605 \times 605 \times 249,792 \times 792 \times 307$ and $1161 \times 1161 \times 416$ respectively. Figure 2 shows the thrust at these resolutions, together with the average experimental result while Table 1 shows the average thrust obtained by experiment and simulations. The comparison between the experimental and numerical thrust improves as the grid resolution increases. Figure 3 shows isosurfaces plotted at $\mathrm{Q}$ criterion $=2$, superimposed with pressure contour at time $=$ $0.12 \mathrm{~T}$ for different grid resolutions. We observe that as resolution increases, the isosurfaces increases due to having more number of grid cells. However, at $d x=0.024$, there is much less isosurfaces as compared to $d x=0.018$ and 0.012 .

We next move on to the acoustic analysis at different grid resolutions. The sensitivity of CFD grid resolution on acoustic results has been studied for the baseline case. Further, the effect of different control surfaces on the overall sound pressure level has been studied to determine the use of appropriate permeable control surface for subsequent analyses of serrated propellers. Figure 4 shows two types of fictitious control surfaces namely, CS_0 (cylinder without end cap), CS_1 (cylinder with end caps) employed in the present study which are located at a distance of $1.1 \mathrm{R}$ ( $\mathrm{R}$ is the radius of the propeller) from the centre of the propeller. Figure 5 shows the observer point locations at which the acoustic results will be monitored. The control surfaces are discretized into 42467 and 53044 triangular panels respectively, with finer discretization near the downstream end to enable accurate representation of acoustic sources, especially the contribution from tip vortices. The reason for studying the two surface types is to understand the effect of end caps (i.e. closure) on acoustic prediction. The use of open surface avoids wake penetrating the downstream end cap. The quadrupole source term in the porous FW-H equation has been neglected, since the control surface is assumed to reasonably contain the non-linear sound sources within it. Also, given that the propeller speed is subsonic, the effect of non-linear source terms is weaker in the far-field. However, they will be predominant when the observer point is located closer to the propeller axis of rotation, in the downstream end due to contributions from the tip vortices.

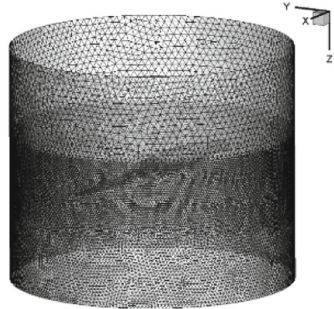

(a)

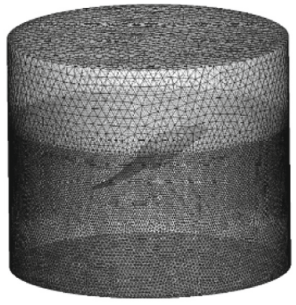

(b)

Fig. 4. Geometry and mesh of fictitious control surface located at $1.1 \mathrm{R}$ from the center of propeller (a) CS_0 (b) CS_1. 


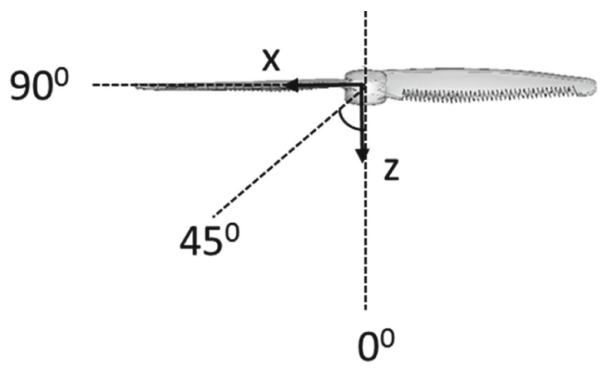

Fig. 5. Locations of observer points.

Table 2. Effect of CFD grid on acoustic results.

\begin{tabular}{l|l|l|l|l}
\hline Control surface & Observer position & \multicolumn{3}{|l}{ OASPL $(\mathrm{dBA})$} \\
\cline { 3 - 5 } & & A $(0.024)$ & $\mathrm{B}(0.018)$ & $\mathrm{C}(0.012)$ \\
\hline \multirow{3}{*}{ CS_0 } & $0^{0}$ & 67.68 & 65.85 & 66.51 \\
\cline { 2 - 5 } & 30 & 67.85 & 66.44 & 66.54 \\
\cline { 2 - 5 } & 45 & 68.26 & 66.07 & 66.74 \\
\cline { 2 - 5 } & 60 & 68.15 & 65.86 & 66.25 \\
\cline { 2 - 5 } & 90 & 67.83 & 66.33 & 66.22 \\
\hline CS_1 & 0 & 70.77 & 71.18 & 72.11 \\
\cline { 2 - 5 } & 30 & 70.24 & 69.92 & 70.27 \\
\cline { 2 - 5 } & 45 & 70.13 & 70.12 & 69.68 \\
\cline { 2 - 5 } & 60 & 70.37 & 70.10 & 70.16 \\
\cline { 2 - 5 } & 90 & 69.38 & 69.54 & 69.06 \\
\hline
\end{tabular}

From Table 2, it can be observed that grid B furnishes acoustic results that are closer to the fine grid resolution $\mathrm{C}$. Hence, from these analyses, we decide that the minimum grid length of $0.018 c$ will be used for all simulations in this study. Running a case for one period in parallel using 960 Intel(R) Xeon(R) CPU E5-2690 v3 @ $2.60 \mathrm{GHz}$ processors takes about $70 \mathrm{~h}$. As for the acoustic part, we used a maximum of 8 processors in parallel for computing the acoustic results at 8 observer locations which took about $4 \mathrm{~h}$.

\section{Experimental Setup}

The acoustic and thrust measurements are conducted inside the anechoic chamber (located at the Temasek Laboratories@National University of Singapore). The propeller is mounted on the ATI mini40 Load Cell SI-20-1, which provides the thrust measurement, and the microphones are mounted at Points 1-5. The five points are aligned along a circle of radius, $R=600 \mathrm{~mm}$, with Point 1 directly beneath the propeller and Point 5 directly above the propeller. The rest of the Points 2, 3 and 4 are spaced out equally along the circumference of the circle at $45^{\circ}$ angle between each 
point as seen in Fig. 6. The acoustics and thrust measurements of the 5030 propeller will be taken at rotation speeds of $9000 \mathrm{rpm}$, for validation against the numerical data.

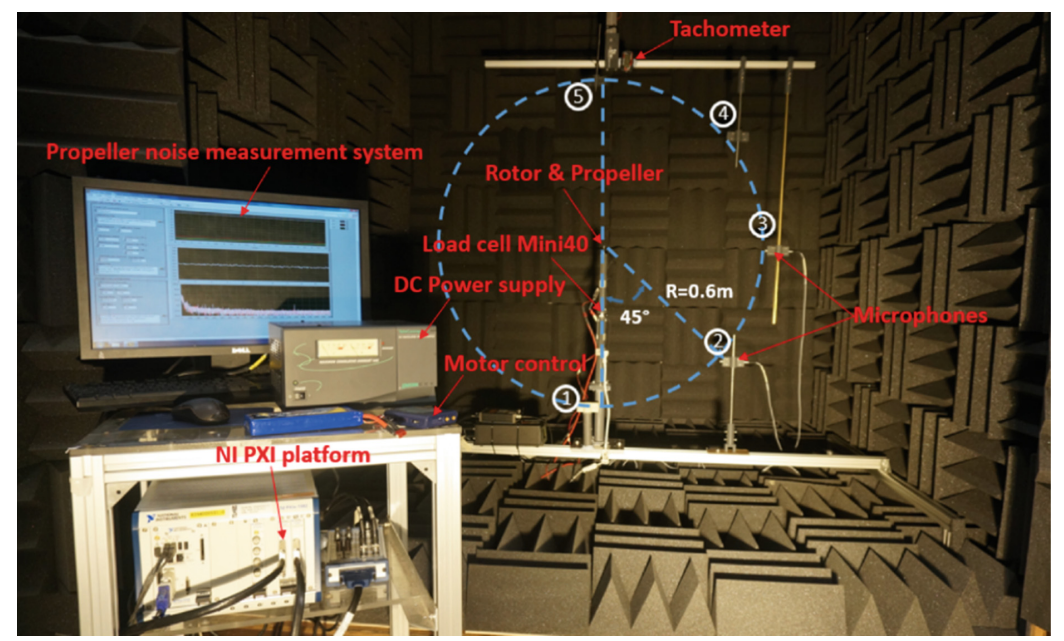

Fig. 6. Schematic of the experimental setup inside the anechoic chamber

The microphones (Brüel \& Kjær Model 4953 1/2 inch condenser microphone) are connected to a preamplifier and signal conditioner (Brüel \& Kjær Model 2669, and NEXUS 2690-A, respectively). The analog signal of the microphone was sampled at $f_{s}=100 \mathrm{kHz}$ by a fast analog-to-digital board (National Instruments PXI 6221). Each recording consists of $10^{6}$ samples.

To avoid aliasing, a Butterworth filter was used to low-pass filter the signals at $f_{L P}=0.499 f_{s}-1(49,899 \mathrm{~Hz})$. The corresponding power spectrograms were computed using a short-time Fourier transform providing a spectral resolution of about $0.1 \mathrm{~Hz}$. Using the microphone sensitivity and accounting for the amplifier gain setting, the voltage power spectrograms were converted to the power spectrograms of $p^{\prime} / p_{\text {ref }}$, where $p^{\prime}$ is the fluctuating acoustic pressure and $p_{\text {ref }}=20 \mu \mathrm{Pa}$ is the commonly used reference pressure. Converted to decibels and time averaged, these become sound pressure level spectra $S P L(f)$, where $f$ is the measured frequency. An A-weighting correction was applied to the SPL spectra to account for the relative loudness perceived by the human ear. The corresponding overall sound pressure level (OASPL) is obtained by integrating the SPL spectra:

$$
O A S P L=10 \log _{10} \int_{0}^{f_{\text {upper }}} 10^{0.1 S P L(f)} d f,
$$

where $f_{\text {upper }}$ is the highest frequency of interest which in this study is $10 \mathrm{kHz}$.

The thrust generated by the propeller is measured by an ATI mini40 load cell SI20-1 whose force range and accuracy in the measured direction ( $\mathrm{Z}$ direction) are $60 \mathrm{~N}$ 
( $\approx 6000 \mathrm{~g})$ and $\pm 0.01 \mathrm{~N}(\approx 1 \mathrm{~g})$, respectively. The analog signal of the load cell was sampled at $f_{s}=5 \mathrm{kHz}$ by a fast analog-to-digital board (National Instruments PXI $6221)$. Each recording consists of $5 \times 10^{4}$ samples, the recorded signal is filtered with a low-pass filter at $f_{L P}=20 \mathrm{~Hz}$ and then the mean value of the filtered data is calculated as the thrust of propeller. A tachometer is used to measure rotational speed of the propeller.

\section{Methodology}

The objectives of the present study are to preliminarily explore the serrated biopropeller concept using numerical modelling and further develop a low noise biopropeller design strategy which can be used to optimize the propeller's blade geometry of the small $(<20 \mathrm{~cm})$ quadcopter. The general steps of our methodology are:

1. Selection of a baseline propeller for our current study and measurement of its thrust and acoustic performance experimentally.

2. Use of our in-house numerical aerodynamic and acoustic solver to perform validation.

3. Re-design the propeller by adding serration to the its blades using CAD software and perform simulations to evaluate the performance of propellers with different serration parameters.

\subsection{Initial Baseline Propeller Selection and Serrated, Cut-off Propeller Design}

As mentioned earlier, our objective is to reduce the noise signature due to the propellers of small quadcopters weighing around 250-350 g. Hence, in this study, we have chosen the 5030 propeller as our baseline case. Each propeller can provide a thrust of around 80 to $90 \mathrm{~g}$, rotating at $9000 \mathrm{rpm}$, and this give a total thrust of 320-360 g.

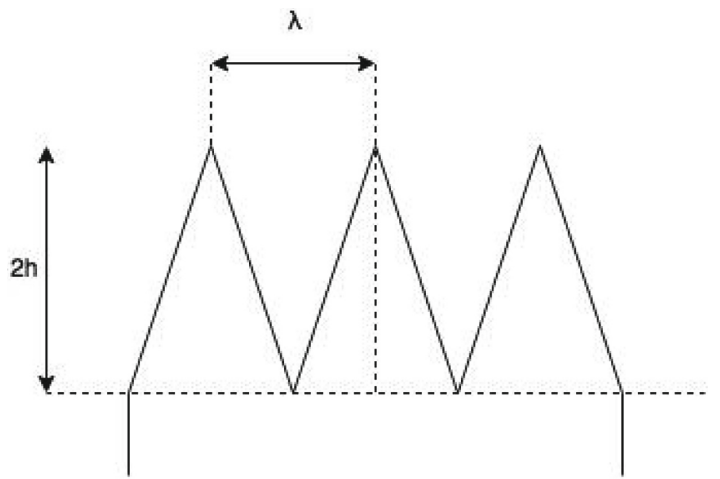

Fig. 7. Schematics of saw tooth serration parameters. 
Next, we move on to the serrated propeller design. The saw tooth serrated design is represented in Fig. 7. The height of each saw tooth is $=2 h$ and the distance between each saw tooth peak is $\lambda$. In accordance with other references [21], the key parameter often used in literature is the ratio of $\lambda / h$. In this study, we fix $h$ while varying the value of $\lambda$. The values $\lambda / h$ selected are given in Table 3 .

Table 3. Range of $\lambda / h$ selected

$$
\begin{array}{l|l|l|l|l|l}
\hline \lambda / h & 0.5 & 0.75 & 1 & 1.25 & 2 \\
\hline
\end{array}
$$

In the current design, part of the blade material is removed to create the serrations. This is different from the method used by some other studies [21], whereby the serrations are added onto the blades of the propeller. Due to the reduction in the surface area of the blades, it would not be fair to simply compare the baseline with the serrated propellers, even when using force coefficients which takes into account the surface area. Hence, a special type of propeller known as the cut-off propeller is created, as shown in Fig. 8. It has approximately the same surface area as the serrated propellers. One concern is that this modification changes the profile of the propeller's blade. However, this is inevitable because the adding of serrations modifies the propeller blade's profile as well. Hence, we will be comparing the serrated propellers with the baseline and cut-off propellers for a more comprehensive analysis.

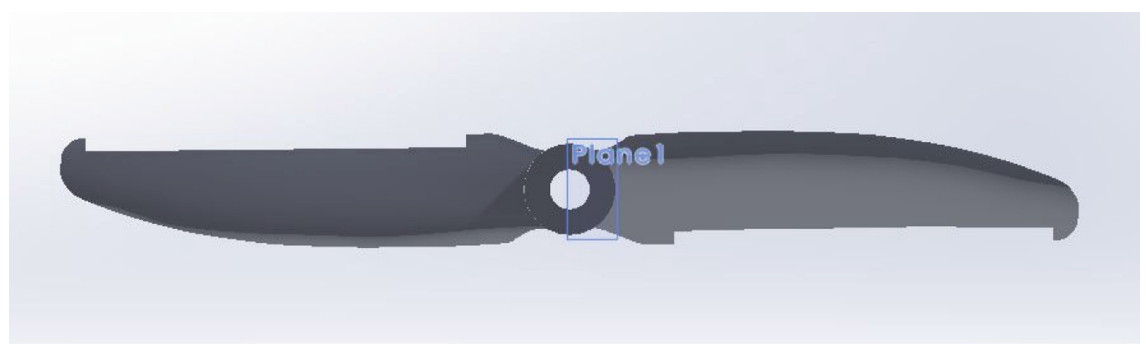

Fig. 8. Frontal CAD view of the cut-off propeller.

\section{Results and Discussions}

\subsection{Force Comparison}

Figure 9 shows the thrust of the propellers over one period while Table 4 shows the average thrust. The experimental result is also given for comparison. Due to cost and time constraint, only two of the better performing serrated propellers have been $3 \mathrm{D}$ printed for validations. 


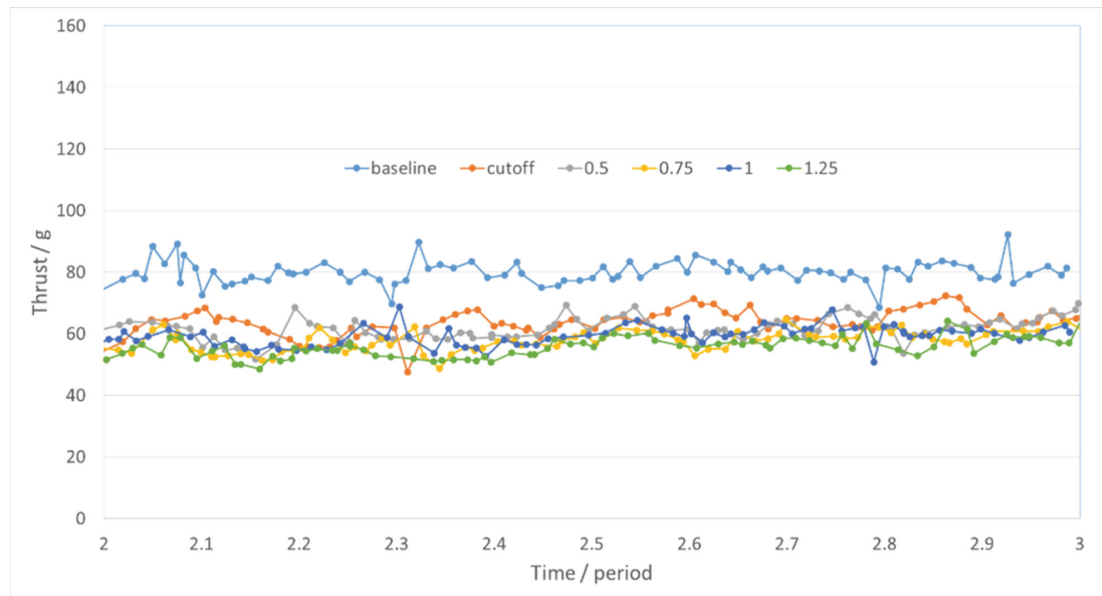

Fig. 9. Thrust generated by different propellers over one period.

Table 4. Surface area and average thrust generated by the different propellers

\begin{tabular}{|c|c|c|c|c|c|c|}
\hline & Baseline & Cut-off & $\lambda / h=0.5$ & $\lambda / h=0.75$ & $\lambda / h=1$ & $\lambda / h=1.25$ \\
\hline Surface area $/ \mathrm{cm}^{2}$ & 15.6 & 13.6 & 13.6 & 13.6 & 13.4 & 13.6 \\
\hline Average numerical thrust/g & 80.4 & 67.4 & 65.6 & 61.6 & 62.5 & 59.5 \\
\hline
\end{tabular}

Comparing between the surface area of the baseline and cut-off propellers, there is a $12.8 \%$ decrease in surface area. The serrated propellers have similar surface areas as the cut-off propeller. The average thrust of the cut-off propeller is $16.2 \%$ lower than that of the baseline case. Hence, the drop in thrust is higher than the surface area. However, we must also understand that the cut-off is simply a shortcut alternative to compare between serrated and unserrated propellers of similar area. It is not an aerodynamically ideal design and therefore will generate a lower than expected thrust. Moreover, thrust increase or decrease is usually exponential, instead of linear.

If we compare the cut-off propeller with the serrated ones, we observe that there can be a drop or increase in the thrust, although the surface areas of these propellers are similar. These vary from -2.7 to $-11.7 \%$.

\subsection{Flow Visualizations}

We now turned our attention to the comparison of the baseline, cut-off and serrated propellers. The $\lambda / h=1$ serrated propeller is chosen since it gives the highest thrust. Similar to the previous comparison, there is only minor difference in the surface pressure distribution on the propellers. The key differences in this case lies in the vortex shedding at the trailing edge. As shown on the circled regions in Fig. 10, the serrated 
propeller tends to produce elongated, narrow and long vortices. It has been mentioned in some papers that the serration breaks up the larger vortices into smaller ones, and this in turn reduces the noise level of the propeller. This is because larger vortices are more energetic and they created larger pressure fluctuations during shedding.

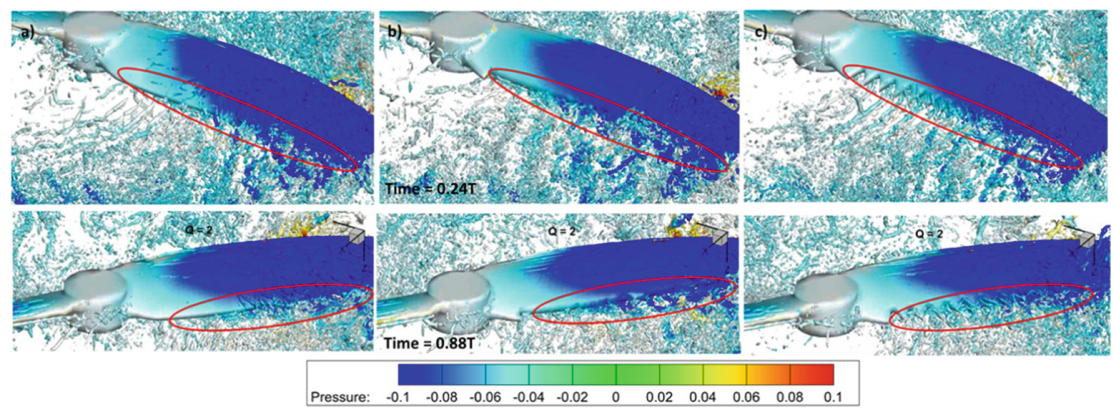

Fig. 10. Isosurface plotted at $Q$ criterion of 2, superimposed with pressure contour of the a) baseline, b) cut-off and c) $\lambda / \mathrm{h}=1$ propellers at time $=0.24 \mathrm{~T}$ and $0.88 \mathrm{~T}$. Circle regions denote differences.

\subsection{Acoustic Analysis}

We now present the results for propellers with various serrated trailing edge configurations. Table 5 presents the OASPL values at an observer distance of $10 \mathrm{R}$ from the propeller hub. In all the cases, the height of serration is fixed while the amplitude of serrations is varied to study the influence of trailing edge serrations on the acoustic field. Owing to computational time, the CFD results are extracted for one cycle after steady state convergence is achieved, followed by acoustic analysis. Figure 11 presents the plot of overall sound pressure level (dBA) for various serrated configurations at various observer locations. In general, the effect of including serrations at the trailing edge reduced noise level, especially in the vicinity of the downstream end. As evidenced in the isosurface plots in Fig. 10, the propeller with $(\lambda / h=1)$ reduces the intensity of trailing edge vortices compared to baseline and cut-off propeller configurations. As they are convected downstream, reduced noise levels are perceived near downstream observer locations. This is also reflected in OASPL plot in Fig. 11 and Table 5. However, the role of serrations in reducing noise levels are not effective for the in-plane observer point and its immediate vicinity. This supports the fact that dipole sources resulting from oscillating surface pressure distribution on the propeller are the main sources of noise at these locations. Furthermore, based on numerical investigations, there seems to be an optimal serrated configuration corresponding to $(\lambda / h=1)$ which can reduce downstream noise levels from 2.3 to nearly $5 \mathrm{dBA}$. Further numerical investigations will be conducted in future to substantiate the above fact. The amplitude and spacing of serrations play a crucial role in controlling the intensity of the 
shed vortices, especially those shed from the blunt roots of the serrations. This is possibly one of the reasons why the noise levels begin to increase beyond an optimal spacing of serrations [22, 23]. For instance, the noise levels begin to increase for $\lambda / h<0.75$. Therefore, the effect of introducing serrations at the trailing edge eventually results in lower noise levels by enhancing the bypass transition to turbulence, compared to conventional transition to turbulence through laminar boundary layer.

Table 5. Comparison of OASPL values (dBA) at various locations for baseline and serrated 5030 propellers at $9000 \mathrm{rpm}$.

\begin{tabular}{|c|c|c|c|c|}
\hline Propeller & Angle & CS_0 & CS_1 & Experiment \\
\hline \multirow[t]{5}{*}{ Baseline } & 0 & 65.85 & 71.18 & 67.17 \\
\hline & 30 & 66.44 & 69.92 & - \\
\hline & 45 & 66.07 & 70.12 & 67.68 \\
\hline & 60 & 65.86 & - & - \\
\hline & 90 & 66.33 & - & 65.21 \\
\hline \multirow[t]{5}{*}{ Cut-off } & 0 & 67.78 & 71.23 & 68.49 \\
\hline & 30 & 67.03 & 70.28 & \\
\hline & 45 & 65.85 & 69.31 & 69.37 \\
\hline & 60 & 65.05 & - & \\
\hline & 90 & 65.71 & - & 66.36 \\
\hline \multirow[t]{5}{*}{$\mathrm{SR}-1.25(\lambda / h=1.25)$} & 0 & 66.92 & 68.73 & \\
\hline & 30 & 67.20 & 70.30 & \\
\hline & 45 & 66.60 & 70.54 & \\
\hline & 60 & 66.43 & - & \\
\hline & 90 & 65.30 & - & \\
\hline \multirow[t]{5}{*}{ SR $-1(\lambda / h=1)$} & 0 & 65.63 & 65.65 & 68.94 \\
\hline & 30 & 67.12 & 66.52 & \\
\hline & 45 & 67.57 & 67.56 & 67.33 \\
\hline & 60 & 66.57 & - & \\
\hline & 90 & 64.94 & - & 63.48 \\
\hline \multirow[t]{5}{*}{$\mathrm{SR}-0.75(\lambda / h=0.75)$} & 0 & 66.13 & 69.25 & 69.87 \\
\hline & 30 & 65.79 & 68.65 & \\
\hline & 45 & 66.54 & 68.39 & 67.42 \\
\hline & 60 & 67.15 & - & \\
\hline & 90 & 66.15 & - & 66.04 \\
\hline \multirow[t]{5}{*}{$\mathrm{SR}-0.5(\lambda / h=0.5)$} & 0 & 65.94 & 68.97 & \\
\hline & 30 & 66.62 & 70.45 & \\
\hline & 45 & 65.42 & 69.88 & \\
\hline & 60 & 65.87 & - & \\
\hline & 90 & 66.26 & - & \\
\hline
\end{tabular}




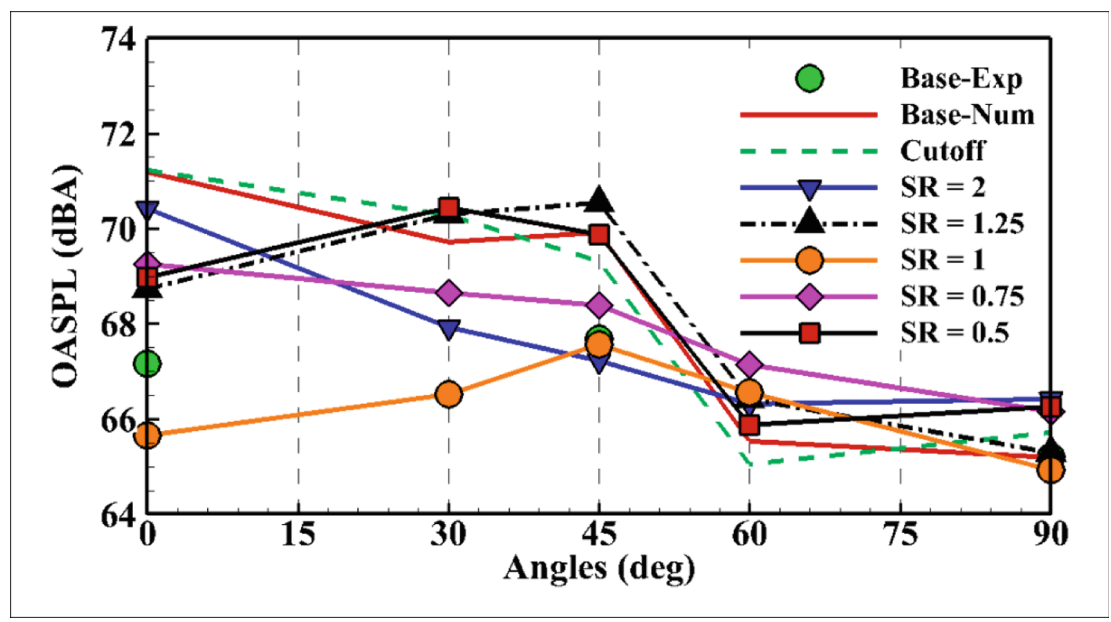

Fig. 11. Comparison of OASPL values for various propeller types.

\section{Conclusions and Recommendations}

Results show that when the serrated propellers are compared to the cut-off propeller, it is a decrease of $2.7 \%$ to $11.7 \%$. There is a general trend of lower acoustic noise. The optimum case lies in the $\lambda / h=1$ case, whereby while there is only a small change in the thrust, it can have up to $5 \mathrm{~dB}$ decrease in acoustic noise. These results demonstrated that serrations can be used to lower the noise level of propeller. More importantly, we have created a computational framework that links the numerical solver to the acoustic solver (based on FWH methodology) to study acoustic performance of propellers. This will be very useful for the systematic testing of future bio-mimetic propeller designs.

For the aero-acoustic part, the results show that the present solver can capture the tonal frequencies occurring at the harmonics of the blade passage frequency. The broadband components of the sound spectrum associated with small scale turbulent velocity fluctuations cannot be captured since the CFD solver is based on an incompressible flow averaged Navier Stokes equation.

\section{References}

1. Gur, O., Rosen, A.: Design of quiet propeller for an electric mini unmanned air vehicle. J. Propul. Power 25, 717-728 (2009). https://doi.org/10.2514/1.38814

2. Sato, S.: Design and characterization of hover nano air vehicle (HNAV) propulsion system (2009). https://doi.org/10.2514/6.2009-3962

3. Agarwal, N.: Study of the unsteady aerodynamics associated with a cycloidally rotating blade (2017)

4. Clark, I.A., et al.: Bio-inspired canopies for the reduction of roughness noise. J. Sound Vib. 385, 33-54 (2016). https://doi.org/10.1016/j.jsv.2016.08.027 
5. Geyer, T., Sarradj, E., Fritzsche, C.: Nature-inspired porous airfoils for sound reduction. In: Tropea, C., Bleckmann, H. (eds.) Nature-Inspired Fluid Mechanics. NNFMMD, vol. 119, pp. 355-370. Springer, Heidelberg (2012). https://doi.org/10.1007/978-3-642-28302-4_21

6. Barone, M.F.: Survey of techniques for reduction of wind turbine blade trailing edge noise (2011)

7. Mittal, R., Iaccarino, G.: Immersed boundary methods. Annu. Rev. Fluid Mech. 37, 239-261 (2005). https://doi.org/10.1146/annurev.fluid.37.061903.175743

8. Williams, J.E.F., Hawkings, D.L.: Sound generation by turbulence and surfaces in arbitrary motion. Philos. Trans. Roy. Soc. A Math. Phys. Eng. Sci. 264, 321-342 (1969). https://doi. org/10.1098/rsta.1969.0031

9. Tay, W.B., Deng, S., van Oudheusden, B.W., Bijl, H.: Validation of immersed boundary method for the numerical simulation of flapping wing flight. Comput. Fluids 115, 226-242 (2015). https://doi.org/10.1016/j.compfluid.2015.04.009

10. Hirt, C.W., Amsden, A.A., Cook, J.L.: An arbitrary lagrangian-eulerian computing method for all flow speeds. J. Comput. Phys. 135, 203-216 (1997). https://doi.org/10.1006/jcph. 1997.5702

11. Yang, J., Balaras, E.: An embedded-boundary formulation for large-eddy simulation of turbulent flows interacting with moving boundaries. J. Comput. Phys. 215, 12-40 (2006). https://doi.org/10.1016/j.jcp.2005.10.035

12. Kim, J., Kim, D., Choi, H.: An immersed-boundary finite-volume method for simulations of flow in complex geometries. J. Comput. Phys. 171, 132-150 (2001). https://doi.org/10.1006/ jcph.2001.6778

13. Liao, C.-C., Chang, Y.-W., Lin, C.-A., McDonough, J.M.: Simulating flows with moving rigid boundary using immersed-boundary method. Comput. Fluids 39, 152-167 (2010). https://doi.org/10.1016/j.compfluid.2009.07.011

14. Kim, D., Choi, H.: A second-order time-accurate finite volume method for unsteady incompressible flow on hybrid unstructured grids. J. Comput. Phys. 162, 411-428 (2000). https://doi.org/10.1006/jcph.2000.6546

15. Balay, S., Gropp, W.D., McInnes, L.C., Smith, B.F.: Efficient management of parallelism in object oriented numerical software libraries. In: Arge, E., Bruaset, A.M., Langtangen, H. P. (eds.) Modern Software Tools in Scientific Computing, pp. 163-202 (1997). https://doi. org/10.1007/978-1-4612-1986-6_8

16. Falgout, R.D., Jones, J.E., Yang, U.M.: The design and implementation of hyper, a library of parallel high performance preconditioners. In: Bruaset, A.M., Tveito, A. (eds.) Numerical Solution of Partial Differential Equations on Parallel Computers, pp. 267-294. Springer, Heidelberg (2006). https://doi.org/10.1007/3-540-31619-1_8

17. Calderon, D.E., Wang, Z., Gursul, I.: Lift enhancement of a rectangular wing undergoing a small amplitude plunging motion. In: 48th AIAA Aerospace Sciences Meeting, Orlando, Florida, pp. 1-18 (2010)

18. Lua, K.B., Lim, T.T., Yeo, K.S.: Scaling of aerodynamic forces of three-dimensional flapping wings. AIAA J. 52, 1095-1101 (2014). https://doi.org/10.2514/1.J052730

19. Brentner, K.S., Lyrintzis, A., Koutsavdis, E.K.: A Comparison of computational aeroacoustic prediction methods for transonic rotor noise. In: American Helicopter Society 52nd Annual Forum (1996)

20. Vieira, A., Lau, F., Mortágua, J.P., Cruz, L., Santos, R.: A new computational tool for noise prediction of rotating surfaces (fact) (2015). https://doi.org/10.5281/ZENODO.1099577

21. Ning, Z., Hu, H.: An Experimental study on the aerodynamic and aeroacoustic performances of a bio-inspired UAV propeller. In: 54th AIAA Aerospace Sciences Meeting, pp. 1-19 (2016). https://doi.org/10.2514/6.2017-3747 
22. Parchen, R., Hoffmans, W., Gordner, A., Bran, K.A.: Reduction of airfoil self-noise at low mach number with a serrated trailing edge. In: International Congress on Sound and Vibration (1999)

23. Gruber, M.: Aerofoil noise reduction by edge treatments (2012)

Open Access This chapter is licensed under the terms of the Creative Commons Attribution 4.0 International License (http://creativecommons.org/licenses/by/4.0/), which permits use, sharing, adaptation, distribution and reproduction in any medium or format, as long as you give appropriate credit to the original author(s) and the source, provide a link to the Creative Commons license and indicate if changes were made.

The images or other third party material in this chapter are included in the chapter's Creative Commons license, unless indicated otherwise in a credit line to the material. If material is not included in the chapter's Creative Commons license and your intended use is not permitted by statutory regulation or exceeds the permitted use, you will need to obtain permission directly from the copyright holder.

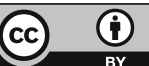

